\title{
Reduction of the Outgassing Segments and LWR Improvement for the Next Generation EUV Lithography
}

\author{
Seiya Masuda, Shinji Tarutani, Sou Kamimura, Shuuji Hirano, Wataru Hoshino, \\ Kazuyoshi Mizutani \\ $R \& D$ management headquarter, Electronic Material Research Laboratories, \\ FUJIFILM Corporation \\ Kawashiri 4000, Yoshida-cho, Haibara-gun, Shizuoka, 421-0396, Japan \\ TEL: +81-548-32-7312, FAX: +81-548-32-3185
}

\begin{abstract}
The continuous studies for both the outgassing reduction and the sensitivity improvement by applying low outgassing photo acid generator with a various kinds of polymer protection group were discussed in this paper. Further reduction of the outgassing segments from the resist was demonstrated to achieve the total outgassing amount below the detection limit of GC-MS (ca. less than $1 \times 10^{10}$ molecules $/ \mathrm{cm}^{2}$ ). Loading a large sized acetal group could be successfully reduced the amount of the outgassing segments from polymer below the tool detection limit, which would be acceptable for a high volume manufacturing tool usage. The development properties of poly(hydroxyl styrene) (PHS) based bulky acetal polymers were measured by changing molecular weight. The high dissolution rate contrast was obtained with the bulky acetel protected low molecular weight polymer. A resolution capability study was carried out with micro exposure tool (MET) at LBNL and Albany. The correlation between LWR through $\mathrm{CD}$ and DOF was measured by loading various amounts of quencher. The resolution capability of newly developed EUV resist had been successfully improved by modifying both resist polymer matrix and quencher amount optimization. It was possible to obtain $27.7 \mathrm{~nm}$ lines with MET tool, where $L W R$ value at $35 \mathrm{~nm} \mathrm{~L} / \mathrm{S}$ was $3.9 \mathrm{~nm}$ with reasonable sensitivity range.
\end{abstract}

Keywords: EUV lithography, chemical amplified resist, outgassing, LWR

\section{Introduction}

EUV lithography is the one of the candidate for half-pitch $32 \mathrm{~nm}$ generation device manufacturing and beyond. In case of EUV lithography, it is necessary to reduce the outgassing segments from the resist during EUV exposure to avoid optics contamination. Watanabe et., al discussed about the outgassing amount by using ESCAP type resist. Both the solvent and decomposition of the acrylate gives a high amount of the outgassing during EUV exposure ${ }^{1}$. Typical ESCAP's outgassing amount was reported by Kim Dean et. $\mathrm{al}^{2}$, which was $3.9 \mathrm{E}+13 \mathrm{molecules} / \mathrm{cm}^{2}$. In our previous paper, we have reported that the most outgassing segments from our bulky acetal type resist was come from sulfonium type photo acid generator (PAG). The outgassing segments from PAG could be reduced by loading substituent group on benzene ring to increase the size of decomposed species from the PAG cation group. The total amount of the outgassing was from 1 to 2 $\times 10^{12}$ molecules $/ \mathrm{cm}^{2}$ that was acceptable range for MET exposure at that time. In this study, we have focused on the further outgassing reduction, preferably to achieve much more less than $1 \times 10^{10}$ molecules $/ \mathrm{cm}^{2}$, which is below GC-MS detection limit.

As $193 \mathrm{~nm}$ immersion technology could be extended to $32 \mathrm{~nm}$ HP with high NA exposure tool. The resolution capability requirement for EUV resist was moved down to $22 \mathrm{~nm}$ HP and below, which is challenging target to satisfy high sensitivity, high resolution capability and small line width roughness (LWR) at the same time. 
In $32 \mathrm{~nm}$ HP generation, LWR value should be 1.7 cause within resist component to reduce LWR is still unclear issue. Fukuda discussed the line edge roughness (LER) in terms of acid concentration, acid diffusion length, molecular size, protection ratio and variation, and image condition using inhouse simulation ${ }^{5}$. All of those parameters may be affected to LER variation in small feature sizes. The latent acid image blur after EUV/EB irradiation can be theoretically explained by Kozawa's acid generation model ${ }^{6}$, where minimum acid blur would be related with the distance from the ionization and acid generation point, such as 2 $-3 \mathrm{~nm}$ initially, that could be indicate the limit of minimizing LER/LWR value as the e-beam irradiation induced acid image blur.

The generated acid migration control could be key parameter to improve resolution and exposure latitude ${ }^{7}$. The increasing amount of quencher against PAG could minimize the acid migration that clearly reduces LER/LWR, however, high amount of base loading gives a low resist sensitivity ${ }^{8,9}$.

The development property induced LER/LWR could be realized by measuring resist surface roughness at half exposed area ${ }^{11,12,13}$, thus surface roughness could be well correlated with the material origin LER/LWR after development

The molecular size of polymer is also one of the important factors to improve LWR and resolution. A small polymer grain size would give a LER/LWR reduction, if the resist system had high acid concentration and low acid diffusion length, which was calculated by simulation ${ }^{5,10}$. However, in case of the low PAG concentration with large acid migration, the large size polymer grain gives a better LER/LWR in both simulation result 5,10 and experimental results with EUV exposure $^{11}$.

Our approaches to reduce the LWR are; a) loading high sensitive PAG with high sensitive polymer platform ${ }^{3}$, b) low molecular weight polymer to down size dissolution segments having high dissolution contrast, and c) minimizing acid diffusion length by loading relatively large amount of base to maintain good latent acid images for the small line feature size.

\section{Experimental}

The amount of the outgassing segments had measured with GC-MS method using synchrotron ring at University Wisconsin under SEMATECH's $\mathrm{nm}$, indicated in ITRS 2005 roadmap. The root EUV program ${ }^{2}$. The sample has been exposed 2 times of Eth energy and repeating 3 wafers exposure, then the outgassing species were corrected into the one thermal desorption tube. The total quantity of the outgassing segments (molecules $/ \mathrm{cm}^{2}$ ) has been determined by calibrated toluene concentration, which was loaded inside of the sample chamber during EUV exposure as the external standard of GC-MS.

To determine the acetal size effect on the outgassing amount, the Van der Waars (VDW) volume of the deprotection group was calculated with CAChe (FUJITSU) software.

As we described in our previous paper ${ }^{3}$, the key resist components such as acetal protected poly(hydroxyl-styrene) (PHS) type polymer and low outgassing PAG were synthesized by ourselves. The synthetic method to incorporate desired acetal protections into PHS-based polymer was written in our previous paper ${ }^{14,15}$

Fractionated polymer was prepared with silica gel column chromatography with the mixture of ethylacetate and chloroform as the eluent.

The resist samples were prepared by mixing the acetal-protected polymers, low outgassing PAG, and organic amine for the quencher into PGMEA solution. The formulated resist solution was coated on HMDS primary treated bare $\mathrm{Si}$ wafer by applying Prebake (PB) at $120^{\circ} \mathrm{C}$ for 90 seconds to obtain $80-125 \mathrm{~nm}$ film thickness. The resist coated wafer was exposed by E-beam writer HL-750 (Vacc. $=50 \mathrm{keV}$, Hitachi) or EUV Micro Exposure Tool (MET, NA $=0.3$, Exitech). Then exposed wafers was subsequently applying Post Exposure Bake (PEB) at $110^{\circ} \mathrm{C}$ for 90 seconds and the resist pattern was developed by FHD- $5^{\circledR}$ developer for 60 sec. (TMAH 2.38\% aq., FUJIFILM Electronic Materials Co., ltd)

The dissolution rate of the acetal polymers were measured with RDA ${ }^{\circledast}$ (Lithotech Japan Co., ltd) by exposing open frame E-Beam exposure, done by $\mathrm{HL}-750^{\boxplus}$ (Hitachi) at $50 \mathrm{keV}$. The surface roughness at the half exposed area was measured with AFM Dimension $3100^{\circledR}$ (Veeco) by scanning $1 \mu \mathrm{m} \times 1 \mu \mathrm{m}$ of the exposed area after development.

LWR value was basically measured with $S-$ $9380^{\circ}$ CD-SEM with rectangle scan mode where the measurement gate console could cover more than $2000 \mathrm{~nm}$ length of the line in one time image $\operatorname{scan}^{16}$. Beside, the patterned wafer with MET@ ALS (LBNL) was 4 inch size, so that $L W R$ value 
was measured by taking top-view images with S$4800^{\circledast}$ (SEM microscope, Hitachi) by 5 times repeating steps in long length direction to cover $2000 \mathrm{~nm}$ length. Then the scanned line pattern images were transferred to off-line CD-SEM measurement software (Hitachi) under the same LWR measurement algorithm with CD-SEM.

\section{Results and Discussion}

\subsection{Reduction of outgassing segments}

As described in the previous paper $^{3}$, we have successfully reduced outgassing segments which was mainly come from PAG, where the total outgassing amount from resist was $1-2 \times 10^{12}$ molecules $/ \mathrm{cm}^{2}$ range. However, it gave small amount of the outgassing segments from acetal protection and solvent (Fig.1) at that time.

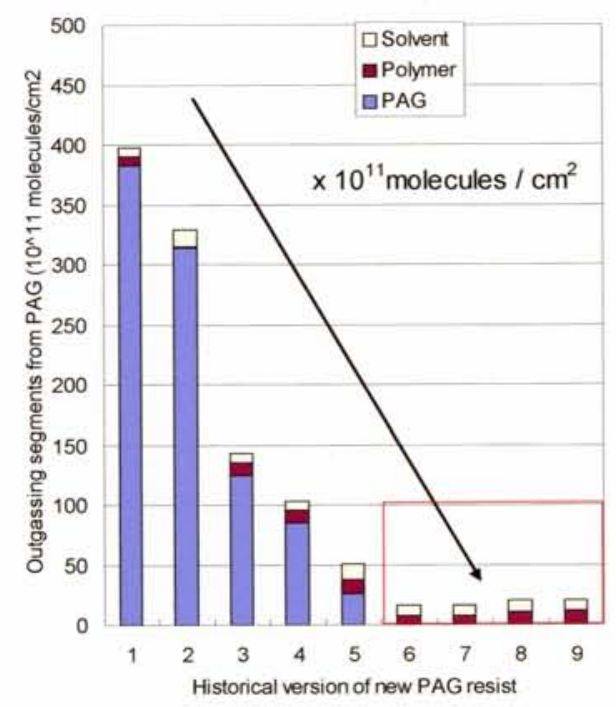

Figure 1 Historical improvement of the resist outgassing

Various kinds bulky acetal group have synthesized to make an effective protection into polymer matrix for the outgassing reduction. The outgassing amount (molecules $/ \mathrm{cm}^{2}$ ) vs. VDW volume of the acetal groups were plotted in Fig. 2 . It was clear that the enlarging the size of acetal group was influenced on the reduction of the outgassing segments from polymer, which could be minimized the outgassing segments below GCMS detection limit.

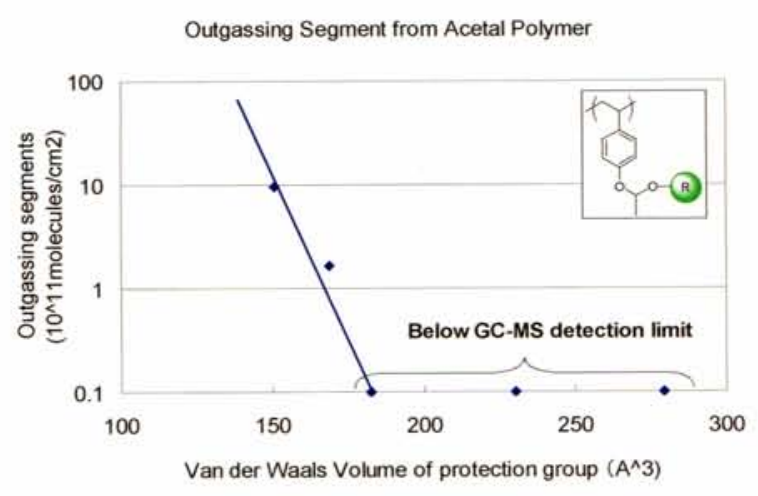

Figure 2 Protection group size dependence on the amount of outgassing segment from polymer

Using bulky acetal group and low outgassing PAG, we have demonstrated GC-MS outgassing measurement with UW method. The results were shown in Fig. 3, as a comparison of previous version of acetal polymer A and ESCAP type polymer $\mathrm{E}$ with a low outgassing PAG.

The newly developed bulky acetal group could be minimized the total amount of the outgassing segments from resist. That was down to $4.5 \times 10^{10}$ molecules $/ \mathrm{cm}^{2}$ from polymer C type resist, and nothing detected from polymer $\mathrm{D}$ type resist, respectively (Fig.3). The interesting thing was observed within the solvent outgassing, which was remained a low level in that case. The solvent might be less volatile from the film if the deprotected acetal group was stayed within the resist film under the high vacuum condition.

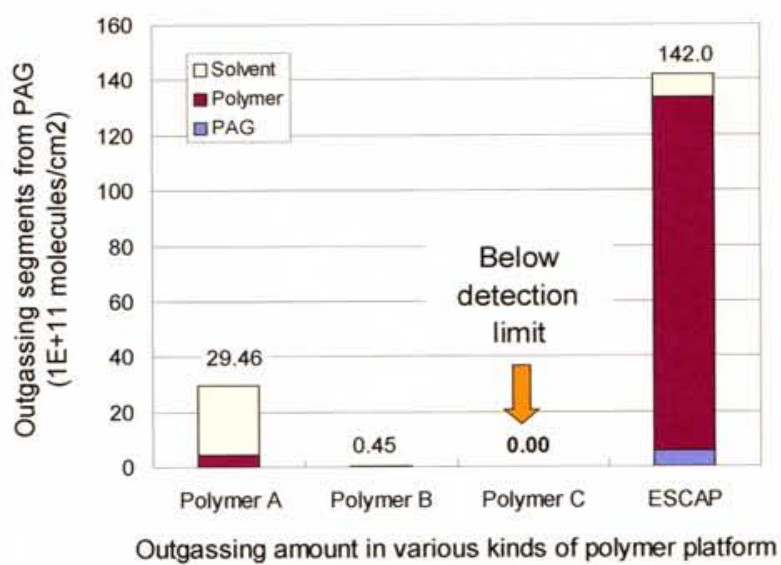

Figure 3 Minimizing outgassing level with newly developed acetal polymer platform

When we compared with the typical ESCAP polymer $E$ with our low outgassing PAG, it gave a relatively high amount of deprotection segments which was come from methacrylate group. The total amount of the outgassing of out 
typical ESCAP resist was $1.42 \times 10^{13}$ molecules / $\mathrm{cm}^{2}$.

It could be said that the bulky acetal polymer platform showed better outgassing property than ESCAP type system at EUV irradiation under the high vacuum condition.

\subsection{LWR improvement study}

\subsubsection{AFM surface roughness study}

The polymer dissolution uniformity could be key parameter for LWR improvement. To clarify the roughness caused by polymer dissolution characteristic, the surface roughness at half-exposed area within those resists were measure by AFM with open frame e-beam exposure.

The maximum roughness was observed at half exposed area in the range of $30-40 \%$ of normalized remaining film thickness (Fig. 4).

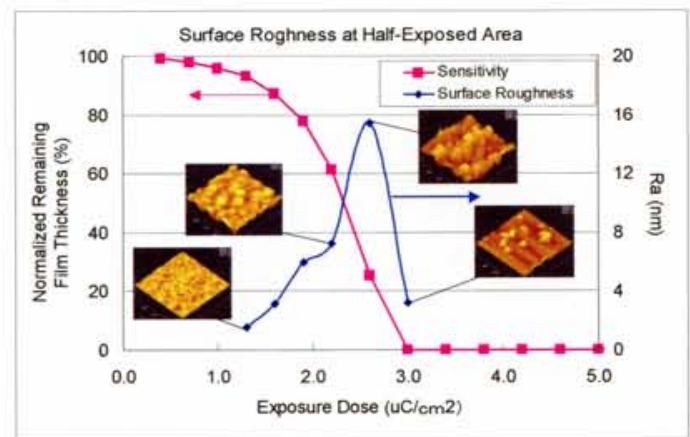

Figure 4 Surface roughness ( $\mathrm{Ra}$ ) variation at half exposed area

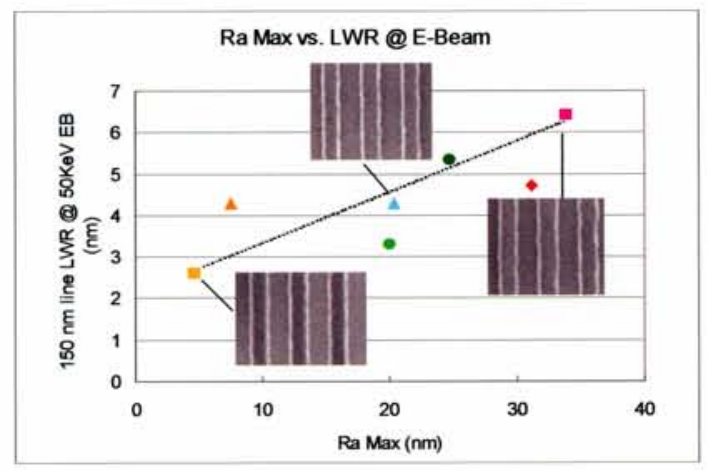

Figure 5 Surface roughness (Ra) vs. $150 \mathrm{~nm}$ LWR with $50 \mathrm{keV}$ E-Beam exposure

The surface roughness $(\mathrm{Ra})$ value at halfexposed area could be roughly predicted the side wall roughness of the patterned line after development. The Ra value at half exposed area varied with $150 \mathrm{~nm}$ LWR with E-beam (Fig. 5).

\subsubsection{Polymer Protection uniformity}

The protection and deprotection uniformity induced surface roughness was studied by fractionating the acetal polymer with column chromatography. It was found that the original polymer was the mixture of the various acetal protected polymer having different dissolution rate. The weight ratio of the fractioned polymer and dissolution rate of the each segments were shown in Fig. 6. The dissolution rate diversity was over $1000 \mathrm{~nm} / \mathrm{sec}$, that might cause a surface roughness during the development.

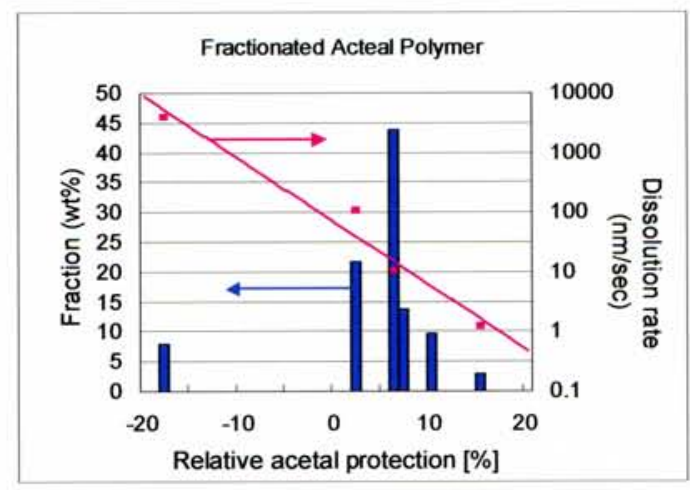

Figure 6 Relative protection ratio against ratio of polymer fraction having various dissolution rate.

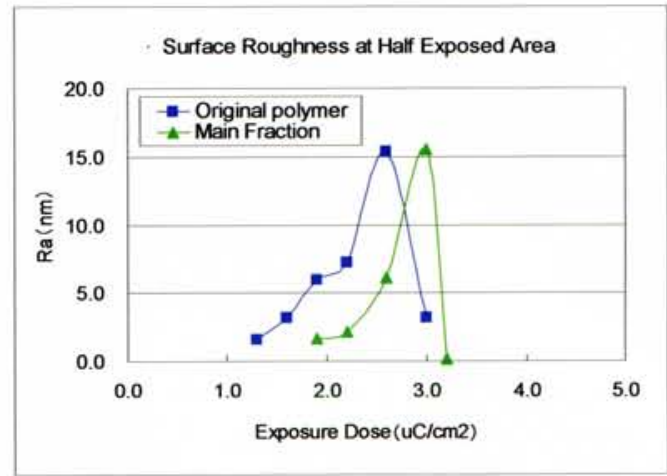

Figure 7 Surface roughness comparison between the original polymer and main fraction polymer resist.

AFM surface roughness measurement was carried out by comparing the original polymer resist and fractioned polymer one which contains only the main fraction having the same acetal protection ratio (Fig. 6). The fractioned polymer should have much more uniform protection rate in the resist film. However, there was no significant difference between two resists (Fig. 7). It was suggested that deprotection diversity inside of polymer could be minor factor for the material induced roughness in case of the acetal type resist. 


\subsubsection{Polymer size effect - Development property}

The size of polymer aggregation may affect to the development property and resolution. A small sized development pixels may enhance both resolution and $\mathrm{LWR}^{5,10}$. The dissolution rate contrast was measured by changing molecular weight $(\mathrm{Mw})$ with acetal polymer type $\mathrm{A}$. The result was shown in Fig. 8, that the lower $\mathrm{Mw}$ polymer gives the better dissolution contrast.

As the low molecular weight polymer caused the high dissolution rate contrast, which might influence the development uniformity of the resist matrix ${ }^{5,10}$. The surface roughness at halfexposed area within those resists were measure by AFM with open frame e-beam exposure.

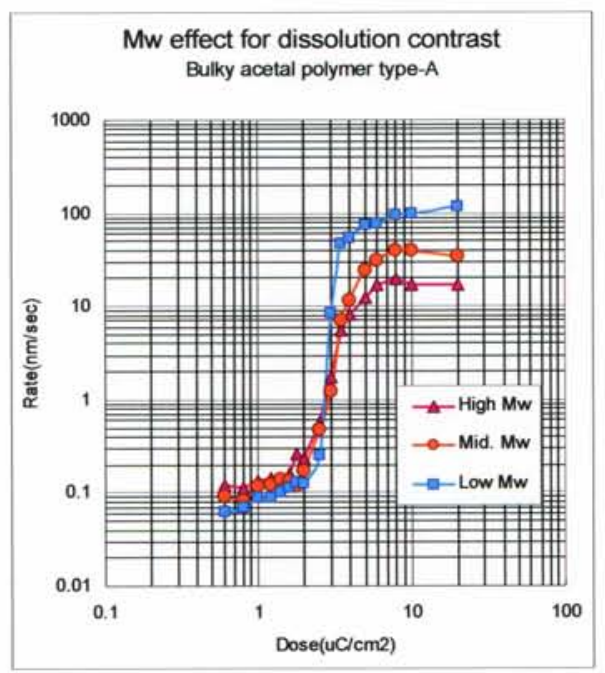

Figure 8 Dissolution rate curves in various kinds of molecular weight acetal polymers type A.

Fig. 9 shows the maximum $\mathrm{Ra}$ value through EB dose by changing polymer molecular weight. We could not see any clear difference between high and low molecular weight of polymers. All of those resists supposed to have the similar development uniformity at the bulk surface. It was suggested that the polymer size induced resist dissolution contrast did not have an impact on this bulk surface roughness, because that was not the same development geometry in the narrow sized space area, such as $60 \mathrm{~nm}$ pitch size.

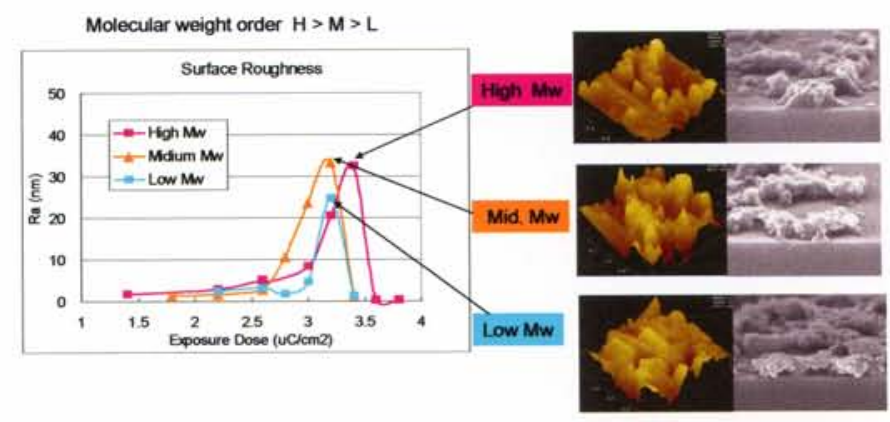

Figure 9 Surface roughness at half exposed area by e-beam exposure

\subsubsection{Polymer size effect - Resolution}

As increasing the size of the dissolution contrast by changing polymer molecular weight, the resist resolution was slightly improved, which result is shown in Fig. 10. The linear resolution capabilities were measured within various kinds of molecular weight polymers exposed at CD 70nm just sized energy with EUV. The high $\mathrm{Mw}$ polymer type resist had only $60 \mathrm{~nm}$ of the linear resolution, although the low Mw one gave $45 \mathrm{~nm}$ of linear resolution under the same EUV exposure condition.

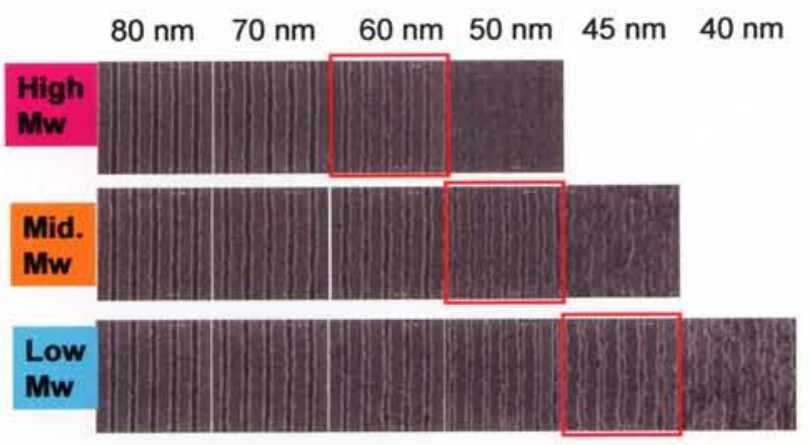

Figure 10 Resolution capability dependence on polymer molecular weight

When we plotted LWR against line size with those 3 resists, LWR value was getting worse by increasing the molecular weight of polymer at small feature sizes (Figure 11). This LWR variation may be related with the polymer's dissolution rate contrast, which influenced on resist resolution limit. The small sized development segments at exposed area could be also affected to this resolution improvement. So that resolution improvement is the one of the key parameters to improve LWR. 


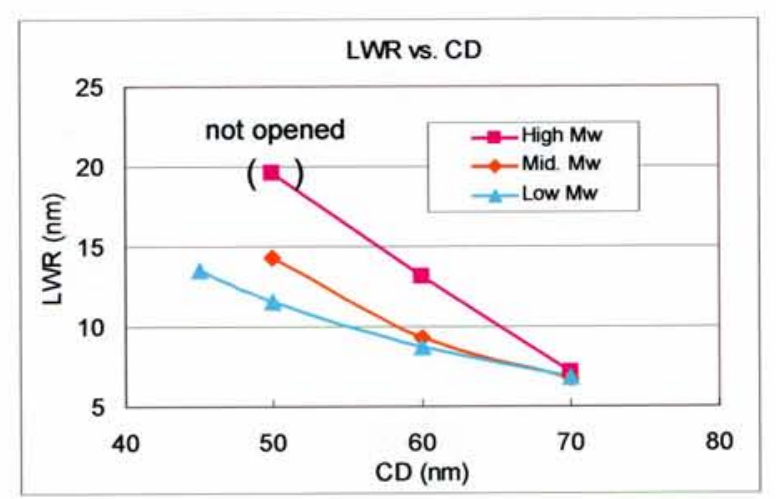

Figure 11 LWR vs. line size in various kinds of polymer $\mathrm{Mw}$

\subsubsection{Loading amount of base - CD vs. LWR}

The acid diffusion control is also important parameters for resolution improvement $^{5,10}$. A relatively high amount of base loading enables to improve a resolution capability of the resist, but it cause the sensitivity loss at the same time. The loading amount of the base significantly had an effect on LWR through CD size toward the resolution limits down to $30 \mathrm{~nm}$ (Fig.12). By increasing the normalized base amount ratio against PAG, from 1 to 3 times, LWR was slightly improved from $5.2 \mathrm{~nm}$ to $3.9 \mathrm{~nm}$ at 35 $\mathrm{nm}$ line, from $7.9 \mathrm{~nm}$ to $4.8 \mathrm{~nm}$ at $30 \mathrm{~nm}$ line, respectively.

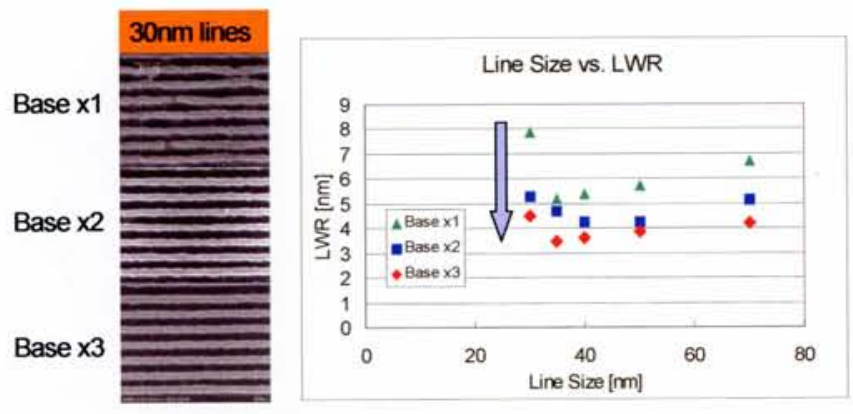

Figure 12 CD vs. LWR by changing loading base amount

\subsubsection{Loading amount of base - DOF vs. LWR}

The other advantage to increase the amount of base was stabilizing the latent acid image contrast caused by normalized image log slope (NILS) degradation. Figure 13 indicated the LWR variation through depth of focus by changing the amount of base.
By increasing the amount of base, the LWR was slightly improved within not only at the best focus points but also defocused area, where NILS was independent on the exposure energy. Minimizing the acid diffusion length would be a key to control the latent acid image contrast, which improves the latitude of LWR through focus.

\subsubsection{Loading amount of base - Sensitivity vs. LWR}

The sensitivity vs. LWR trade-off relationship is very important item to keep high throughput with fine pattern profile for the high volume manufacturing. Basically, the large amounts of base loading cause the sensitivity loss.

So far, we have put an effort on the material designing for both polymer and PAG to obtain a high sensitivity upon EUV irradiation. That gave us a room to add a relatively large amount of base into the resist system with maintaining acceptable range of the resist sensitivity.

A typical trade-off relationship between sensitivity and LWR at $30 \mathrm{~nm}$ lines is shown in Fig. 14. Here LWR value is slightly went up below $20 \mathrm{~mJ} / \mathrm{cm}^{2}$ range. That may cause poor latent acid images in the resist due to shot noise and acid migration. If the EUV scanner's laser power would be applicable to obtain $20-30 \mathrm{~mJ} / \mathrm{cm}^{2}$ of exposure energy, we could enhance the resist material performance in terms of LWR reduction. However, the formulation study was targeted to gain the resist sensitivity around $10-20 \mathrm{~mJ} / \mathrm{cm}^{2}$ range.

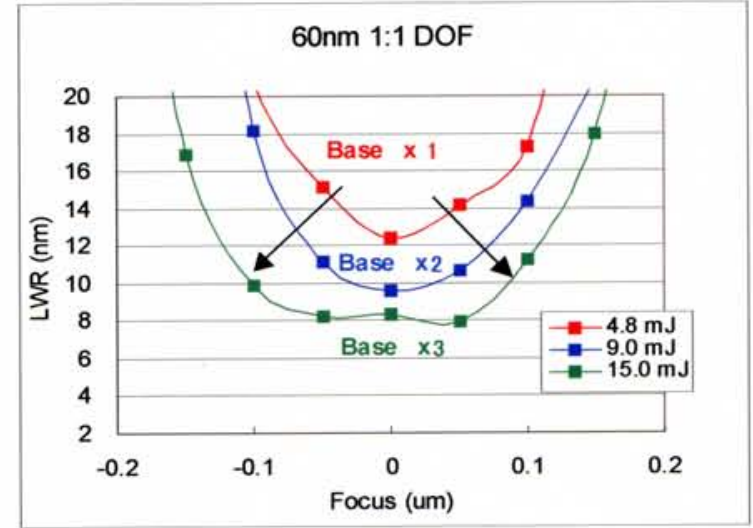

Figure 13 LWR through DOF by changing loading amount of base 


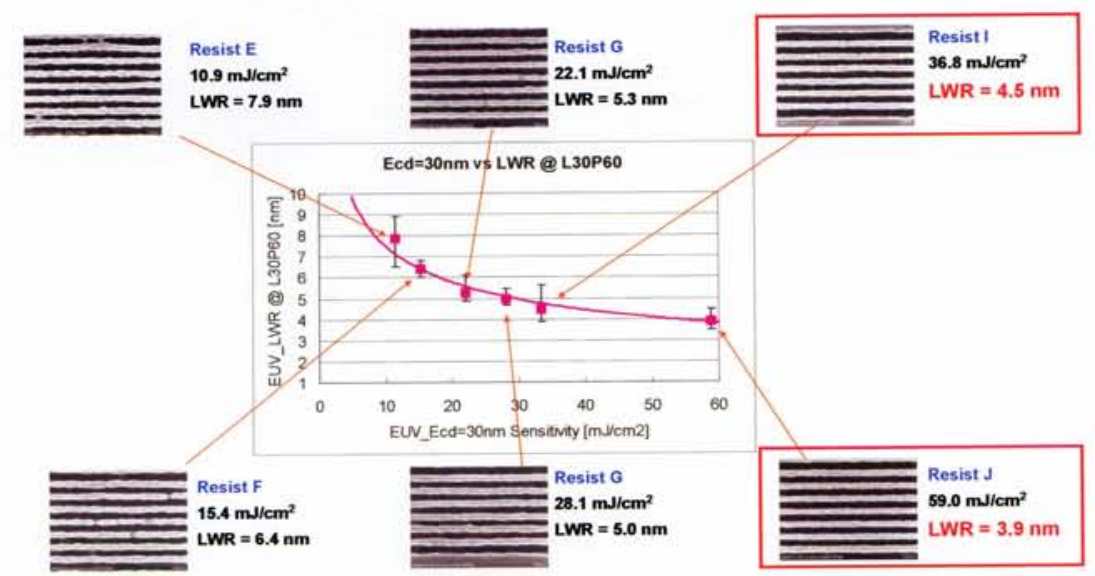

Figure 14 Sensitivity vs. LWR trade-off relationship at $30 \mathrm{~nm}$ dense lines with MET @ ALS

\subsection{Lithographic performance}

The one advantage of acetal based resist chemistry is the low activation energy of the deprotection reaction, that makes resist with very low PEB sensitivity against CD changes. Figure 15 shows $70 \mathrm{~nm}$ CD fluctuations by changing PEB temperature. The PEB sensitivity (PEBS) was $0.246 \mathrm{~nm} /{ }^{\circ} \mathrm{C}$, determined by EUV exposure. That might be acceptable range to obtain good intra wafer CD uniformity for $32 \mathrm{~nm}$ HP generation and below.

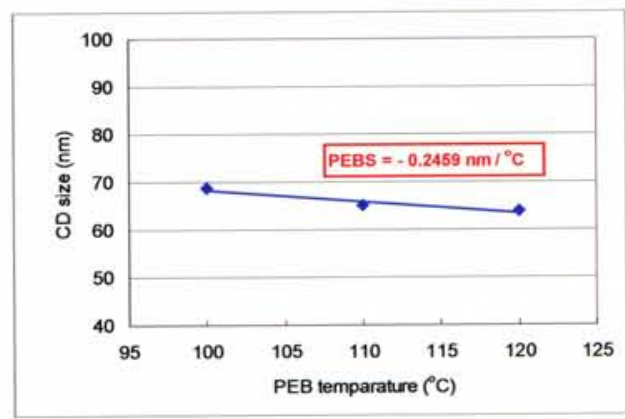

Figure 15 PEB sensitivity of bulky acetal polymer resist

(SEMATECH EUV program)

- MET @ ALS exposure

- Resist K: $18.0 \mathrm{~mJ} / \mathrm{cm}^{2}$, Y-monopole

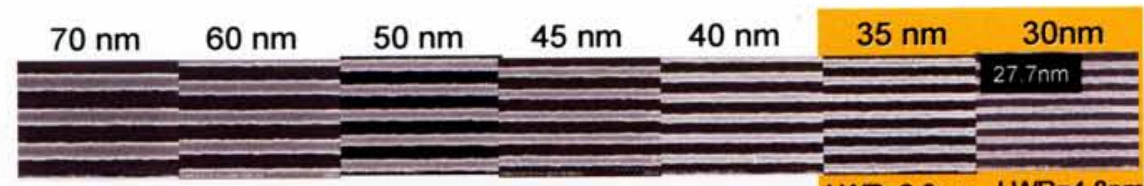

$\mathrm{LWR}=3.9 \mathrm{~nm} \quad \mathrm{LWR}=4.8 \mathrm{~nm}$

- EL 19.3\% @ 35 nm lines

- LWR 3.9 nm @ 35 nm lines

Figure 16 Lithographic performance of the acetal type resist with MET @ ALS

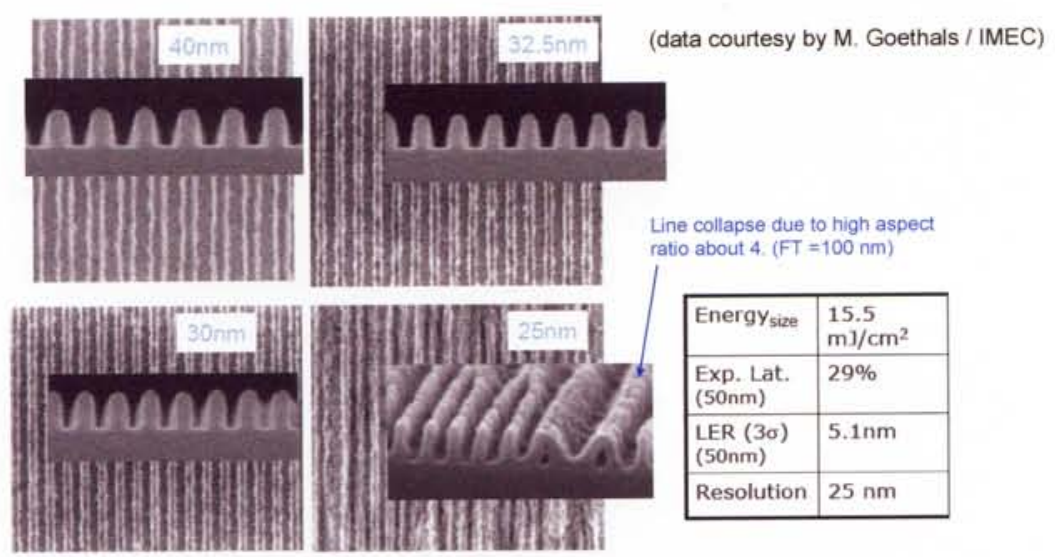

Figure 17 Lithographic performance of the acetal type resist with interference tool at PSI 
The fine pattern profile was obtained by combination of above described all key items into the one resist formulation with EUV dedicated low outgassing PAG (Fig. 16). LWR value was down to $3.9 \mathrm{~nm}$ at $35 \mathrm{~nm}$ line and $4.8 \mathrm{~nm}$ at $30 \mathrm{~nm}$ line, respectively. Sensitivity was around $18 \mathrm{~mJ} / \mathrm{cm}^{2}$ having $19.3 \%$ of the exposure latitude at $35 \mathrm{~nm}$ dense lines.

Ultimate resolution capability had demonstrated with PSI exposure tool (Figure 17). It was possible to resolve $25 \mathrm{~nm}$ lines with interference EUV exposure. Since the film thickness of the resist was around $100 \mathrm{~nm}$ in this study, the line collapse was observed due to high aspect ratio about 4 . We believed that it could be overcome by reducing film thickness to make the line aspect ratio less than 3 .

\section{Conclusion}

Utilizing bulky acetal protection group into the polymer, together with our low outgassing PAG in the resist, it was possible to minimize the outgassing segments from the resist below GC-MS detection limit. LWR variation was measured by changing polymer molecular weight and loading amount of base. The dissolution contrast of the low molecular weight polymer was capable to enhance the resolution limit, which also improved LWR at small line feature size. Addition of relatively large amount base could stabilize acid image contrast in inside of resist that improved LWR through CD size and depth of focus.

About $27.7 \mathrm{~nm}$ dense line resolution capability was observed with MET tool and $25 \mathrm{~nm}$ line 1:1 lines obtained with EUV interference exposure tool.

\section{Acknowledgement}

The author thanks to University of Wisconsin and SEMATECH to measure the outgassing segments from resist. The author also thanks to Dr. Kim Dean and Andrew C. Rudack at SEMATECH Albany to demonstrate EUV exposure, Dr. Mieke Goethals and Dr. Roel Gronheid at IMEC for EUV interference exposure at PSI Switzerland.

\section{References}

1. T. Watanabe, K. Hamamoto, H. Kinoshita, H. Hada and H. Komano: Jpn. J. Appl. Phys.,43 (2004) 3713

2. K. R. Dean , K. E. Gonsalves, and M. Thiyagarajan: Proc. SPIE, 6153, (2006) 61531E

3. S. Masuda, Y. Kawanishi, S. Hirano, S.Kamimura, K. Mizutani, S. Yasunami, and Y. Kawabe: Proc. SPIE, 6153, (2006) 615342

4. D. Van Steenwinckel, J. H. Lammers, L. H. A. Leunissen, and J. A. J. M. Kwinten: Proc. SPIE, 5753, (2005) 269

5. H. Fukuda: Jpn. J. Appl. Phys., 42 (2003) 3748

6. T. Kozawa, K. Okamoto, A. Saeki and S. Tagawa: Jpn. J. Appl. Phys., 44 (2005) 3908

7. D. Van Steenwinckel, J. H. Lammers, T. Koehler, R. L. Brainard, and P. Trefonas: J.Vac. Sci.Technol. B 24 (2006) 316

8. A. R. Pawloski A. Acheta, H. J. Levinson, T. B. Michaelso, A. Jamieson, Y. Nishimura, and C. G. Willson, : J. Microlith., Microfab., Microsyst. 5 (2) (2006) 023001

9. A. Saeki, T. Kozawa, and S. Tagawa, and H. Cao: J. Vac. Sci. Technol B 24 (6) (2006) 3066

10. G. P. Patsis, E. Gogolides and K. Van Werden: Jpn. J. Appl. Phys. 44 (2005) 6341

11. C. A. Cutler, J. F. Mackevich, J. Li, D. J. O'Connell, G. Cardinaleb and R. L. Brainard: Proc. SPIE, 5037 (2003) 406

12. T. Sakamizu, and H. Shiraishi: Proc.SPIE, 5039 (2003) 492

13. N. Kubota, T. Hayashi, T Iwai, H. Komano, and A Kawai: : J. Photopolym. Sci. Technol., (2003) 467

14. S. Malik, A. J. Blackeney, L. Ferreira, B. Maxwell, V.V, Drissche, A. Whewell, T.R. Sarubbi, M. J. Bowden, T.Fujimori, S. Tan, T Aoai, K. Uenishi, K. Kawabe, and T. Kokubo: J. Photopolym. Sci. Technol., 12 (1999) 591

15. T. Fujimori, S. Tan, T. Aoai, F. Nishiyama, T. Yamanaka, M. Momota, S. Kanna, Y. Kawabe, M. Yagihara, T. Kokubo, S. Malik, and L. Ferreira: Proc. SPIE, 3999, (2000) 579

16. A. Yamaguchi, H Fukuda, H Kawada, and T. Iizumi: J. Photopolym. Sci. Technol., 16, (2003) 387 